\title{
Experimental investigation of excited-state lifetimes in atomic ytterbium
}

\author{
C. J. Bowers, D. Budker, E. D. Commins, D. DeMille, S. J. Freedman, A.-T. Nguyen, and S.-Q. Shang \\ Physics Department, University of California, Berkeley, California 94720 \\ and Lawrence Berkeley National Laboratory, Berkeley, California 94720 \\ M. Zolotorev \\ Stanford Linear Accelerator Center, Stanford, California 94309
}

(Received 20 November 1995)

\begin{abstract}
Lifetimes of 21 excited states in atomic $\mathrm{Yb}$ were measured using time-resolved fluorescence detection following pulsed laser excitation. The lifetime of the $4 f^{14} 5 d 6 s^{3} D_{1}$ state, which is of particular importance for a proposed study of parity nonconservation in atoms, was measured to be 380(30) ns.
\end{abstract}

PACS number(s): 32.70.Cs

\section{INTRODUCTION}

This work is motivated by a recent proposal to study atomic parity nonconservation in the $4 f^{14} 6 s^{2}{ }^{1} S_{0}$ $\rightarrow 4 f^{14} 5 d 6 s^{3} D_{1}$ transition in ytterbium (Yb) [1]. This highly forbidden transition is expected to have a relatively large parity nonconserving $E 1$ amplitude due to mixing of the even-parity ${ }^{3} D_{1}$ state with the nearby odd-parity ${ }^{1} P_{1}$ state by the weak interaction [1-3]. A Stark interference measurement of the parity-nonconserving $E 1$ amplitude, similar to those carried out in thallium and cesium [4-6], is being pursued in this laboratory. Since many spectroscopic parameters of the ${ }^{3} D_{1}$ excited state and the forbidden transition from the ground state are unknown, several preliminary measurements are being made to facilitate a measurement of parity-nonconserving effects. Our present measurement of the ${ }^{3} D_{1}$ lifetime is used to estimate the Stark-induced electric-dipole amplitude for this transition.

With our apparatus it was also possible to measure the lifetimes of the $5 d 6 s{ }^{3} D_{2}$ and ${ }^{1} D_{2}$ states, the $6 s 6 p{ }^{3} P_{1}$ state, 16 odd-parity states in the energy range $38000-46000$ $\mathrm{cm}^{-1}$, and an even-parity state at $47584.34 \mathrm{~cm}^{-1}$. Eighteen of the lifetimes were measured, as far as we know, for the first time. Knowledge of these lifetimes is useful for refining the atomic-structure calculations related to the study of parity nonconservation in $\mathrm{Yb}$. A recent critical compilation of previously measured excited-state lifetimes in rare-earth elements can be found in Ref. [7].

\section{EXPERIMENTAL SETUP}

The lifetime measurements were made with the apparatus shown schematically in Fig. 1. The apparatus was essentially the same as that used in previous studies of dysprosium carried out in this laboratory [8]. A simple atomic beam oven appropriate for $\mathrm{Yb}$ was employed here. It consisted of a small tantalum tube $(0.5 \mathrm{~cm}$ diameter $\times 6 \mathrm{~cm}$ long $)$ with one end closed. The oven was resistively heated with tantalum wire coiled inside tubular alumina insulators (not shown in Fig. 1) that surrounded the tantalum tube. A single piece of tantalum foil wrapped around the heaters served as heat shielding. The oven usually operated at about $750 \mathrm{~K}$ (corresponding to a saturated vapor pressure of $\sim 10$ mTorr). The oven was filled with a few grams of metallic $\mathrm{Yb}$, which provided an atomic beam for 10-20 h.

Collimators limited the beam divergence to $\sim 60^{\circ}$ horizontally and $\sim 25^{\circ}$ vertically. At the interaction region $5 \mathrm{~cm}$ from the oven orifice, the atomic density was about $5 \times 10^{10}$ atoms $/ \mathrm{cm}^{3}$. In order to minimize the effect of Zeeman quantum beats on the measured lifetimes, the laboratory magnetic field was shielded with a single 1-mm-thick layer of CO-NETIC high permeability alloy. Holes were cut in the shielding to allow the passage of the atomic beam, laser beams, and fluorescence light.

Three pulsed lasers were used to populate the states here. The frequency-doubled output of a Quanta Ray DCR-2 Nd:YAG laser (where YAG denotes yttrium aluminum garnet) was used to simultaneously pump Quanta Ray PDL-1 and PDL-2 dye lasers. In addition, a fraction of the Nd:YAG light at $1064 \mathrm{~nm}$ could be split off and sent directly to the interaction region. The dye lasers had linewidths of $\sim 0.5$ $\mathrm{cm}^{-1}$ and pulse energies of 2-20 mJ depending on wavelengths. The Nd:YAG laser had a linewidth of $\sim 1.0 \mathrm{~cm}^{-1}$

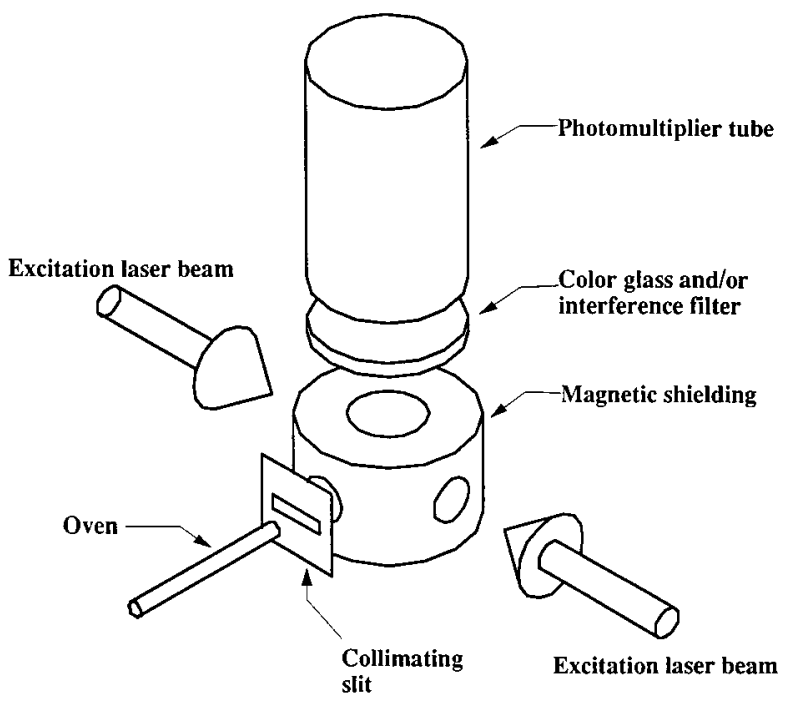

FIG. 1. Schematic drawing of apparatus. Up to three pulsed laser beams overlap with the atomic beam in the center of the apparatus. Fluorescence is detected at $90^{\circ}$ to laser and atomic beams. 


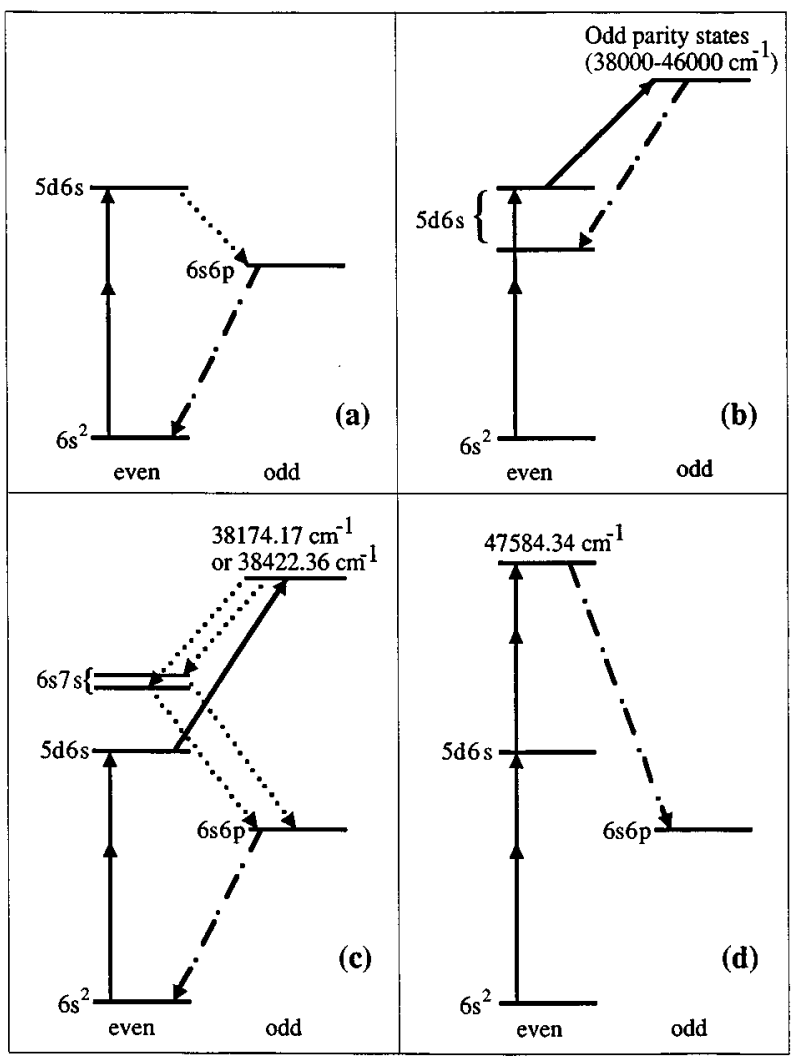

FIG. 2. Excitation and detection schemes. Energy is not to scale. Solid lines represent laser-induced excitation; dotted lines, unobserved fluorescence; dot-dashed lines, observed fluorescence. A solid line with two arrows represents a two-photon transition.

and the portion of energy split off was $\sim 10 \mathrm{~mJ}$ per pulse. The laser system repetition rate was $10 \mathrm{~Hz}$ and the light pulse duration was $\approx 10$ ns. Depending on the excitation scheme employed (Fig. 2), some combination of the three laser beams was directed across the atomic beam. The two dye laser beams propagated in opposite directions perpendicular to the atomic beam, while the Nd:YAG laser beam propagated in the same direction as one of the dye laser beams. Each 2-mm-diam laser beam passed through the same pair of diaphragms to ensure spatial overlap and the path lengths for the beams were adjusted for temporal overlap of the light pulses in the interaction region.

The $4 f^{14} 5 d 6 s^{3} D_{1},{ }^{3} D_{2}$, and ${ }^{1} D_{2}$ states were populated by two-photon transitions from the $4 f^{14} 6 s^{2}{ }^{1} S_{0}$ ground state [Fig. 2(a)]. The ${ }^{1} D_{2}$ state was populated by a two-photon transition with light at $723 \mathrm{~nm}$ from one of the dye lasers. It was not possible to use only one frequency of light to populate the ${ }^{3} D_{1}$ state. From symmetry considerations, it can be shown that the two-photon transition rate from $J_{i}=0$ to $J_{f}=1$ is zero for two degenerate photons. However, the transition rate is nonzero for two sources with different frequencies and polarizations [9]. We used light at $1064 \mathrm{~nm}$ from the $\mathrm{Nd}$ :YAG laser in addition to light at $663 \mathrm{~nm}$ from one of the dye lasers to excite nondegenerate two-photon transitions from the ${ }^{1} S_{0}$ ground state to the ${ }^{3} D_{1}$ state. Although the ${ }^{3} D_{2}$ state can in principle be excited by two degenerate photons from the ${ }^{1} S_{0}$ ground state, in practice it was excited with two different frequencies. Light at 1064 and $651 \mathrm{~nm}$ was prepared in the manner described above. For the nondegenerate two-photon transitions, the fluorescence signal was maximized by rotation of a wave plate (nominally $\lambda / 2$ ) inserted in the dye laser beam. Typically, $\sim 10^{6}-10^{7}$ atoms were excited to either the $5 d 6 s^{3} D_{1},{ }^{3} D_{2}$, or ${ }^{1} D_{2}$ state per laser system pulse.

The second dye laser was used to populate 16 different odd-parity states by electric-dipole transitions from the $5 d 6 s$ states [Figs. 2(b) and 2(c)]. The selectivity of the excitation scheme was ensured by confirming that signals disappeared when any of the excitation beams was blocked. A calibrated dye laser diffraction grating with precision better than $1 \mathrm{~cm}^{-1}$ allowed reliable identification of excited states from published energy-level tables [10,11].

An even-parity level $\left(47584.34 \mathrm{~cm}^{-1}\right)$ was excited by a two-photon transition from the ${ }^{3} D_{2}$ state with light at 1064 and $744 \mathrm{~nm}$ [Fig. 2(d)]. The identification of this transition was confirmed by delaying the light at $744 \mathrm{~nm}$ so that it arrived after the 1064-nm light but before a significant fraction of the ${ }^{3} D_{2}$ state had decayed. The absence of fluorescence light at the appropriate wavelength verified that the second transition required light at both 1064 and $774 \mathrm{~nm}$.

The lifetimes were measured by observing the time dependence of the fluorescence light emitted in the decay of the excited states. A Burle 8850 photomultiplier tube positioned $7 \mathrm{~cm}$ from the interaction region perpendicular to the atomic beam and the laser beam directions detected the fluorescence, as shown in Fig. 1. Color glass and/or interference filters were used to select the appropriate decay channel (see Fig. 2) and block scattered excitation light. The anode current in the phototube was kept below $10 \mathrm{~mA}$ to minimize nonlinearity due to space-charge effects. The photomultiplier output was digitized and averaged over 256 laser pulses by a Tektronix 2430A oscilloscope. The oscilloscope bandwidth was $40 \mathrm{MHz}$ for a single sweep. The effective bandwidth was increased to $150 \mathrm{MHz}$ for some measurements, using a built-in sampling function. The oscilloscope was triggered by the same signal that activated the $Q$ switch of the Nd:YAG laser. Time jitter associated with the trigger was small relative to the time resolution of the oscilloscope.

For many transitions, there was non-negligible background due to scattered excitation light and pickup from the power supply of the Nd:YAG laser by the detection electronics. This background was estimated for each transition by recording the signal with one of the dye lasers tuned 5-10 $\mathrm{cm}^{-1}$ off resonance. This background was subtracted from the corresponding signal on resonance. Data from a measurement of one transition are shown in Fig. 3.

\section{TIME DEPENDENCE OF THE FLUORESCENCE SIGNAL}

For many of the states that we studied, it was possible to observe fluorescence coming directly from the state populated by the laser pulses, as shown in Figs. 2(b) and 2(d). When the excitation light pulse is much shorter than the lifetimes of the states involved and in the absence of any effects of hyperfine structure and external magnetic fields, the time dependence of the fluorescence signal is a simple exponential 


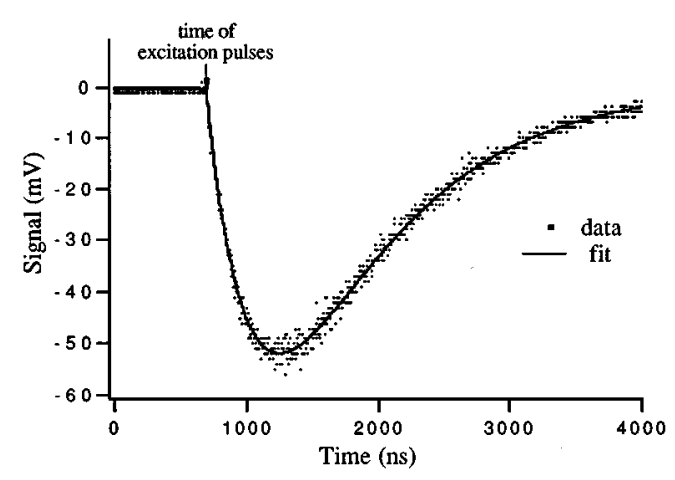

FIG. 3. Fluorescence signal used to extract the lifetimes of the $5 d 6 s^{3} D_{1}$ and the $6 s 6 p^{3} P_{1}$ states. The detection system was sensitive to fluorescence at $556 \mathrm{~nm}$ from the decay of ${ }^{3} P_{1}$ to the ground state. The signal is negative since it was taken directly from the phototube. The data are the difference of on- and off-resonance data sets (see the text), each of which represents an average over 256 consecutive pulses. For the fit shown, the lifetimes are 375 and $880 \mathrm{~ns}$.

$$
S(t) \propto \frac{1}{\tau} \exp (-t / \tau)
$$

where $\tau$ is the natural lifetime of the excited state.

In other cases, direct fluorescence occurred at wavelengths outside the spectral sensitivity of the photomultiplier tube or too near to one of the excitation wavelengths. For these cases, we chose to observe fluorescence from a subsequent cascade [Figs. 2(a) and 2(c)]. Here the signal is influenced by the lifetimes of the intermediate states in the cascade chain. For a cascade involving two excited states [Fig. 2(a)], the time dependence of the fluorescence light emitted with the decay of the second state is given by

$$
S(t) \propto \frac{1}{\tau_{1}-\tau_{2}}\left[\exp \left(-t / \tau_{1}\right)-\exp \left(-t / \tau_{2}\right)\right],
$$

where $\tau_{1}$ and $\tau_{2}$ are the natural lifetimes of the two states involved. Both quantities $\tau_{1}$ and $\tau_{2}$ can be extracted from the time dependence of the signal. However, the time dependence is symmetric with respect to exchange of lifetimes, so there is an ambiguity in the assignment of lifetimes to states. The ambiguity is resolved if one of the lifetimes has been measured independently.

For cascades involving three excited states [Fig. 2(c)], the time dependence of the fluorescence light emitted with the decay of the third state is given by

$$
\begin{aligned}
S(t) \propto & \frac{\tau_{1} \exp \left(-t / \tau_{1}\right)}{\left(\tau_{1}-\tau_{2}\right)\left(\tau_{1}-\tau_{3}\right)}+\frac{\tau_{2} \exp \left(-t / \tau_{2}\right)}{\left(\tau_{2}-\tau_{3}\right)\left(\tau_{2}-\tau_{1}\right)} \\
& +\frac{\tau_{3} \exp \left(-t / \tau_{3}\right)}{\left(\tau_{3}-\tau_{1}\right)\left(\tau_{3}-\tau_{2}\right)}
\end{aligned}
$$

where $\tau_{1}, \tau_{2}$, and $\tau_{3}$ are the natural lifetimes of the three states involved. Again the assignment of measured lifetimes to states requires additional measurements. The independent measurements used to assign states to the lifetimes measured in cascade decays will be discussed at the end of Sec. IV.

\section{RESULTS}

The results of the lifetime measurements are summarized in Table I. The precise energies and dominant configuration and term assignments are those of Refs. [10] and [11]. The excitation and detection schemes correspond to Fig. 2. The wavelength of the detected fluorescence channel(s) is given in the fourth column. The last column contains the results of previous lifetime measurements along with the references.

The lifetimes in this work were extracted by a leastsquares fit of the data with the appropriate time dependence [Eq. (1), (2), or (3)]. For decays involving short lifetimes, these functions were folded with the measured response of the detection system to intentionally scattered pulsed laser light, in order to correct for the excitation pulse duration and the time response of the photomultiplier tube and oscilloscope. Lifetimes extracted from multiple data sets were averaged and the uncertainty was taken to be the spread in the fitted values, which was typically 5-10\%. The source of this spread, which is $\sim 3$ times greater than the statistical uncertainty expected due to shot noise, was not understood. Additional sources of systematic uncertainty that also contributed to the error estimates are discussed below.

Although the fitting procedure corrected for the excitation pulse duration and the time response of the detection apparatus, the correction is only approximate since neither the detector response nor the evolution of the excited state over the duration of the laser pulse was measured directly. The systematic uncertainty in this procedure is a significant component of the overall error estimate for lifetimes shorter than $100 \mathrm{~ns}$.

As discussed in Sec. II, the background was estimated with off-resonance measurements. Nevertheless, variations of the background sources would prevent perfect background subtraction. The effect of this on the extracted lifetimes was estimated by varying the amount of background subtracted from a given data set and refitting the lifetimes. This error was largest for short, weak signals where scattered light from the excitation pulses overlapped with much of the signal. Suppression of scattered light without signal degradation depended on the proximity of the fluorescence wavelength to the excitation wavelengths and the availability of the appropriate color glass and interference filters. The measurement of $\tau\left(45155.33 \mathrm{~cm}^{-1}\right)=25(20) \mathrm{ns}$ had the largest fractional uncertainty due to background.

Several other potential sources of systematic error were considered. For transitions to the ground state, resonant absorption and reemission of the fluorescence light by atoms (radiation trapping) can affect the time dependence of the fluorescence signal. In cascades involving the very strong transition from $6 s 6 p^{1} P_{1}$ to the ground state at $399 \mathrm{~nm}$, the effect of radiation trapping was observed by raising the oven temperature to increase the atomic density (Fig. 4). Normally, the beam density was low enough for radiation trapping to be negligible. As a check, in measurements where fluorescence was observed from the decay of the $6 s 6 p{ }^{3} P_{1}$ state to the ground state, the atomic density was increased by a factor of 10 over normal operating conditions and no significant change in the measured lifetimes was observed.

Due to motion of the atoms in the atomic beam, the time independence of the fluorescence signal can also be affected 
TABLE I. Summary of lifetime measurement results.

\begin{tabular}{|c|c|c|c|c|c|}
\hline Energy $\left(\mathrm{cm}^{-1}\right)$ & $\begin{array}{c}\text { Leading } \\
\text { configuration } \\
\text { and term } \\
\text { assignment }\end{array}$ & $\begin{array}{c}\text { Excitation } \\
\text { and detection } \\
\text { scheme }\end{array}$ & $\begin{array}{l}\lambda \text { fluorescence } \\
\text { detected }(\mathrm{nm})\end{array}$ & $\begin{array}{c}\tau(\mathrm{ns}) \\
\text { this work }\end{array}$ & $\begin{array}{c}\tau(\mathrm{ns}) \\
\text { other references }\end{array}$ \\
\hline \multicolumn{6}{|c|}{ Even-parity states } \\
\hline 24489.102 & $4 f^{14} 5 d 6 s^{3} D_{1}$ & $a$ & 556 & $380(30)$ & \\
\hline 24751.948 & $4 f^{14} 5 d 6 s^{3} D_{2}$ & $a$ & 556 & $460(30)$ & \\
\hline 27677.665 & $4 f^{14} 5 d 6 s^{1} D_{2}$ & $a$ & 399,556 & $6700(500)$ & \\
\hline 47584.34 & $4 f^{13} 5 d 6 s 6 p^{1} P_{1}$ & $d$ & 330,338 & $125(12)$ & \\
\hline \multicolumn{6}{|c|}{ Odd-parity states } \\
\hline 17992.007 & $4 f^{14} 6 s 6 p^{3} P_{1}$ & $a$ & 556 & $850(50)$ & $\begin{array}{l}827(40)^{\mathrm{a}} \\
760(80)^{\mathrm{b}} \\
860(40)^{\mathrm{c}} \\
820(20)^{\mathrm{d}} \\
850(80)^{\mathrm{e}} \\
850(80)^{\mathrm{f}} \\
875(20)^{\mathrm{g}} \\
872(2)^{\mathrm{h}} \\
840^{\mathrm{i}}\end{array}$ \\
\hline 38174.17 & $4 f^{14} 6 s 7 p^{3} P_{1}$ & $c$ & 399 & $120(30)$ & \\
\hline 38422.36 & $4 f^{13} 5 d 6 s^{2}{ }^{3} D_{1}$ & $c$ & 399 & $120(30)$ & \\
\hline 42647.72 & $4 f^{13} 5 d^{2} 6 s^{5} D_{2}$ & $b$ & 551 & $140(13)$ & \\
\hline 42725.78 & $4 f^{14} 5 d 6 p^{3} F_{2}$ & $b$ & 548 & $68(9)$ & \\
\hline 43254.78 & $4 f^{14} 5 f 6 s^{3} F_{3}$ & $b$ & 540 & $88(10)$ & \\
\hline 43297.51 & $4 f^{14} 5 f 6 s^{1} F_{3}$ & $b$ & 539 & $62(9)$ & \\
\hline 43433.85 & $4 f^{14} 5 f 6 s^{3} F_{2}$ & $b$ & 528,535 & $26(7)$ & \\
\hline 43532.77 & $4 f^{13} 5 d^{2} 6 s^{5} D_{1}$ & $b$ & 525,532 & $1820(200)$ & \\
\hline 43659.38 & $4 f^{14} 6 s 8 p^{3} P_{1}$ & $b$ & 522,529 & $140(20)$ & \\
\hline 43805.69 & $4 f^{14} 6 s 8 p^{3} P_{2}$ & $b$ & $518,525,539$ & $140(20)$ & \\
\hline 43815.67 & $4 f^{13} 5 d^{2} 6 s^{5} P_{3}$ & $b$ & 525,539 & $120(12)$ & \\
\hline 44017.60 & $4 f^{14} 6 s 8 p^{1} P_{1}$ & $b$ & 512,519 & $50(20)$ & $\begin{array}{l}39.1(3.5)^{d} \\
47(4)^{f}\end{array}$ \\
\hline 44251.88 & $4 f^{13} 5 d^{2} 6 s^{5} S_{2}$ & $b$ & 513,527 & $450(30)$ & \\
\hline 44453.47 & $4 f^{14} 5 d 6 p^{3} F_{3}$ & $b$ & 508 & $22(7)$ & \\
\hline 45155.33 & $4 f^{14} 5 d 6 p^{1} D_{2}$ & $b$ & 503 & $25(20)$ & $14(1)^{\mathrm{f}}$ \\
\hline 45956.27 & $4 f^{14} 6 s 6 f^{3} F_{2}$ & $b$ & 466,472 & $53(9)$ & \\
\hline${ }^{\mathrm{a}}$ Reference [12]. & & ${ }^{\mathrm{d}}$ Referenc & [15]. & & ${ }^{\mathrm{g}}$ Reference [18]. \\
\hline${ }^{\mathrm{b}}$ Reference [13]. & & ${ }^{\mathrm{e}}$ Referenc & [16]. & & ${ }^{\mathrm{h}}$ Reference [19]. \\
\hline${ }^{\mathrm{c}}$ Reference [14]. & & ${ }^{\mathrm{f}}$ Referenc & [17]. & & ${ }^{\mathrm{i}}$ Reference [20]. \\
\hline
\end{tabular}

by a change in detection efficiency with position. For our geometry, the largest of these effects comes from the dependence of the interference filter transmission on the incidence angle. We estimate this effect to be negligible for all lifetimes measured, amounting to $\leqslant 1 \%$ for the worst case (i.e., the longest-lived state $5 d 6 s^{1} D_{2}$.) Our estimate was verified in measurements in which the excitation beams were moved to different regions of the volume viewed by the phototube, and no significant variations were observed.

Another possible source of systematic error is superfluorescence, which occurs when the density of the excited-state atoms is large. The typical excited-state densities in this experiment were low enough that, given the known decay wavelengths and typical dipole matrix elements, superfluorescence is not expected to occur. As a check, the density of excited atoms was varied by a factor of 10 for measurements in several transitions and no variation in the measured lifetimes was observed.

Systematic deviations can also arise from modulations in the fluorescence light due to precession of the excited atoms in an external magnetic field (Hanle effect or Zeeman quantum beats) [21]. In general, the amplitude of modulation depends on the angular momentum of the states involved and the polarization of the excitation and detected light relative to the magnetic field direction. The modulation frequency depends on the strength of the magnetic field and the excited-state magnetic moments. As shown in Fig. 5, Zeeman quantum beats were observed in the unshielded laboratory magnetic field, $\sim 500 \mathrm{mG}$ oriented at $\sim 45^{\circ}$ from the direction of the observed fluorescence. With magnetic shielding, the field was reduced to $\sim 50 \mathrm{mG}$ at about $10^{\circ}$ of the direction of observation. Data taken with the magnetic 


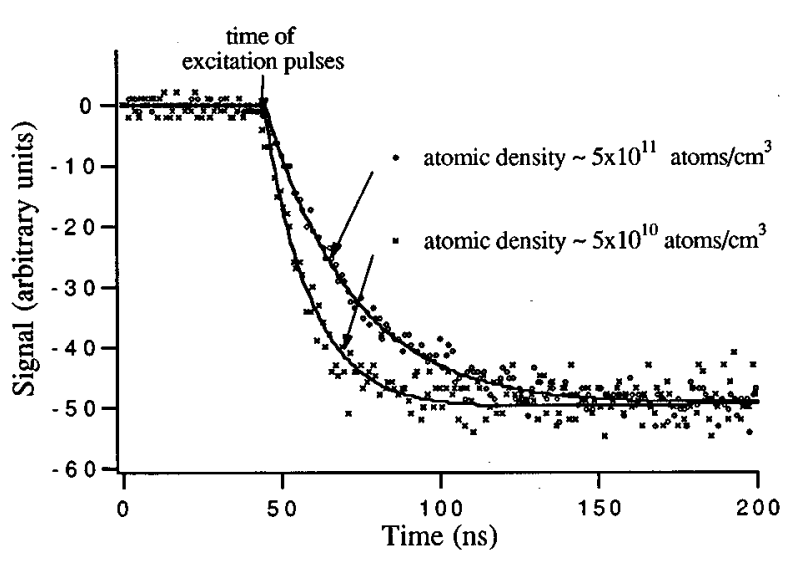

FIG. 4. Fluorescence signals illustrating the effect of radiation trapping. The $5 d 6 s^{1} D_{2}$ state was excited and fluorescence light at $399 \mathrm{~nm}$ was observed from the decay of the $6 s 6 p{ }^{1} P_{1}$ state. The signal taken with a high atomic density shows a longer rise time than the signal taken with the atomic density $\sim 10$ times lower. The relative size of the two signals has been scaled to facilitate a comparison of the rise times.

shielding are well fit with Eq. (1), (2), or (3), with no indication of quantum beats. This is to be expected since the residual field and the size of the magnetic moments involved yield precession periods of $\sim 10 \mu \mathrm{s}$, comparable to the longest lifetime measured. For short lifetimes, the effect of Zeeman quantum beats was negligible. For long lifetimes, it is possible that quantum beat periods about equal to the excited-state lifetime would not be obvious in the data, yet could affect the lifetimes extracted with the fitting procedure. Because the light polarization was not well controlled in this

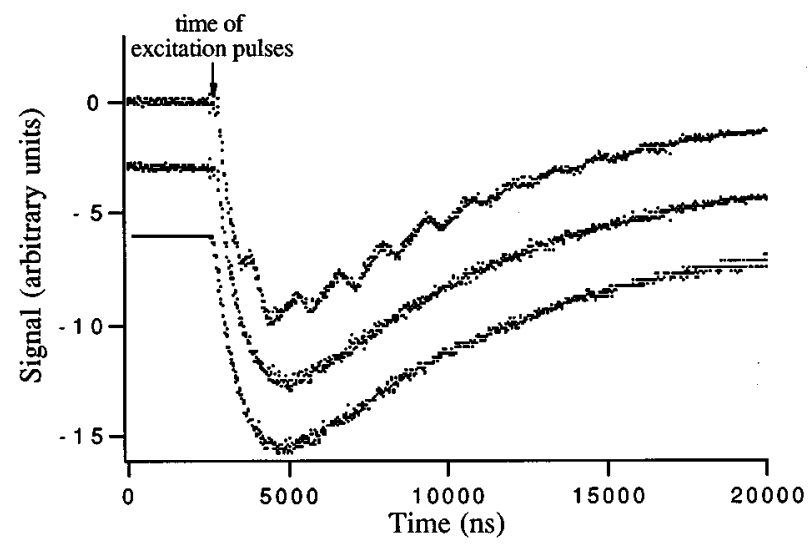

FIG. 5. Three fluorescence signals taken under different magnetic-field conditions. For the purpose of presentation, the traces have been shifted vertically. The $5 d 6 s^{1} D_{2}$ state was excited and fluorescence light at $556 \mathrm{~nm}$ from the decay of the $6 s 6 p^{3} P_{1}$ state was observed. The top trace shows the fluorescence signal when the laboratory magnetic field was not shielded. The middle trace was taken under the same conditions, except that a large, inhomogeneous magnetic field was applied by placing a magnet next to the vacuum chamber. The bottom trace was taken with the laboratory magnetic field shielded as described in the text. The two lower traces exhibit no discernible modulation. Under all three conditions, the fitted lifetimes are consistent within the stated uncertainty. experiment, we estimated the maximum possible effect on the extracted lifetimes due to Zeeman quantum beats in the measured residual magnetic field. Since the axis of precession was inside the angular acceptance of the fluorescence detection, the modulation of the observed fluorescence was reduced. Except for the longest lifetime measured in this work $\left({ }^{1} D_{2}\right)$, the possible effect of Zeeman quantum beats on the measured lifetimes was found to be negligible relative to other sources of experimental error. In the case of the ${ }^{1} D_{2}$ state, the lifetime was extracted from data taken without shielding the laboratory magnetic field. Although fast quantum beats are clearly visible (top trace of Fig. 5), they do not affect the fitted lifetime. This lifetime agreed with that obtained while shielding the laboratory magnetic field.

Modulation of the observed fluorescence intensity can arise in the absence of an external magnetic field from the interaction of electronic and nuclear magnetic moments (hyperfine quantum beats). $\mathrm{Yb}$ has two stable isotopes with nonzero nuclear spin $\left({ }^{171} \mathrm{Yb}, I=\frac{1}{2}, 14.27 \% ;{ }^{173} \mathrm{Yb}, \quad I=\frac{5}{2}\right.$, $16.08 \%$ ), so only $30 \%$ of the total signal can be modulated. The amplitude of the modulation depends on the relative polarizations of the excitation and detected light and the electronic and nuclear angular momenta of the states involved. The modulation frequencies are directly related to the excited-state hyperfine energy splittings. Although the geometry was not optimized for the suppression of hyperfine quantum beats, no evidence of modulation was observed in the absence of an external magnetic field. As with Zeeman quantum beats, one must consider the possibility of modulation periods similar to the lifetime affecting the values extracted from the fits.

The lifetimes of the $5 d 6 s^{3} D_{1},{ }^{3} D_{2}$, and ${ }^{1} D_{2}$ states were measured by observing fluorescence through the $6 s 6{ }^{3} P_{1}$ state [Fig. 2(a)]. The hyperfine splittings of the ${ }^{3} P_{1}$ state have been measured [22] and are all greater than 1 $\mathrm{GHz}$, and estimates of the hyperfine splittings of the $D$ states [23] indicate that the quantum beat frequencies are above the detection bandwidth. Hyperfine quantum beats should therefore be negligible for these measurements.

Many of the high-lying energy levels have mixed configurations, so it is difficult to estimate the hyperfine splittings reliably. Since we cannot be certain that the hyperfine beats do not occur on the same time scale as the lifetimes of these states, we calculated the maximum possible modulation amplitude for each case [24]. We considered the worst case possibility that one of the hyperfine frequencies gives modulations on the same time scale as the excited-state lifetime. Then the maximum effect of this modulation on the extracted lifetime was determined. For all cases, the effect was found to be small relative to other systematic uncertainties.

As discussed in Sec. III, the time dependence of the fluorescence signal from a single cascade decay does not allow unambiguous assignment of lifetimes to states. The lifetime of the $6 s 6 p^{3} P_{1}$ state was measured in three different cascades from the $5 d 6 s^{3} D_{1},{ }^{3} D_{2}$, and ${ }^{1} D_{2}$ states. The common lifetime for the three cascades was identified with the ${ }^{3} P_{1}$ state. Alternatively, independent measurements of the lifetime of the ${ }^{3} P_{1}$ state (see Table I) could be used to resolve the ambiguity, resulting in the same conclusion.

The lifetimes of the odd-parity states at 38174.17 and $38422.36 \mathrm{~cm}^{-1}$ were measured by observing fluorescence 
from the $6 s 6 p{ }^{1} P_{1}$ state [Fig. 3(c)]. The lifetime of the $6 s 6{ }^{1} P_{1}$ state $(\approx 5 \mathrm{~ns})$ was measured previously [7]. There are three possible even-parity intermediate states through which the $6 s 6{ }^{1} P_{1}$ state could have been populated. The lifetimes of these three states were measured previously to be $\tau\left(6 s 7 s^{3} S_{1}\right)=12.5(1.5)$ ns [25] and 15.9(1.9) ns [26], $\tau\left(6 s 7 s^{1} S_{0}\right)=45.8(1.0) \quad \mathrm{ns} \quad[27], \quad$ and $\tau(35196.98$ $\left.\mathrm{cm}^{-1}\right)=1120(50) \mathrm{ns}[20]$. In the present experiment, the fluorescence signal decays completely in less than $300 \mathrm{~ns}$, implying that the state at $35196.98 \mathrm{~cm}^{-1}$ does not significantly contribute to the observed signal. We assume that ${ }^{1} P_{1}$ was populated through some combination of ${ }^{3} S_{1}$ and ${ }^{1} S_{0}$, as shown in Fig. 2(c). Because the ratio of signal to noise was low for these cases, it was not possible to determine the contributions of the two different paths to the observed signal from the fit. The uncertainty in the exact amount from each path is reflected in the uncertainty in the lifetimes of these states.

\section{DISCUSSION}

Three of the lifetimes reported here were measured previously. The $4 f^{14} 6 s 6 p^{3} P_{1}$ lifetime was measured in several previous experiments, as indicated in Table I. Our result agrees with all measurements within the experimental uncertainties. (It should be noted that the uncertainty quoted for the measurement of this lifetime in Ref. [19] is purely statistical.) The short lifetimes of the states at 44017.60 and $45155.33 \mathrm{~cm}^{-1}$ were determined with large uncertainties here compared to those of previous measurements. Within the uncertainties, our results agree with previous measurements. The lifetime of the $6 s 6 p^{1} P_{1}$ state does not appear in Table I. Our observations of decays involving this state are consistent with the previous measurements of $\sim 5 \mathrm{~ns}$ [7]. However, the duration of the excitation light pulse and the time resolution of the detection system preclude a precise measurement for this short lifetime.

Our main goal was the measurement of the $5 d 6 s^{3} D_{1}$ lifetime. The forbidden $6 s^{2}{ }^{1} S_{0} \rightarrow 5 d 6 s^{3} D_{1}$ transition in $\mathrm{Yb}$ lends itself to a Stark interference experiment, in which the parity-nonconserving electric-dipole amplitude interferes with a much larger Stark-induced electric-dipole amplitude produced by an external electric field [1]. We are preparing an experiment to measure the Stark-induced electric-dipole amplitude for this transition. This Stark amplitude can also be estimated using the ${ }^{3} D_{1}$ lifetime. As argued in [1], one factor that determines the Stark-induced amplitude is the dipole matrix element between the $5 d 6 s^{3} D_{1}$ and $6 s 6 p^{3} P_{1}$ states. The ${ }^{3} D_{1}$ state decays predominantly to the ${ }^{3} P_{0,1,2}$ states. In the limit of pure configurations and $L S$ coupling (which is well satisfied for the ${ }^{3} D_{1}$ and ${ }^{3} P_{J}$ states according to [10]), the branching ratio of each of these channels can be obtained by weighting with the appropriate angularmomentum and phase-space factors [28]. This yields a value for the partial decay rate $\Gamma\left({ }^{3} D_{1} \rightarrow{ }^{3} P_{1}\right)$ and hence the dipole matrix element $\left|\left(5 d 6 s^{3} D_{1}|| r|| 5 d 6 p^{3} P_{1}\right)\right|=(2.2 \pm 0.1) a_{0}$.

\section{ACKNOWLEDGMENTS}

The authors wish to thank I. B. Khriplovich and H. Shugart for useful discussions. This research has been supported in part by the U.S. Department of Energy under Contracts Nos. DE-AC03-76SF00098 and W-31-ENG-38 and by the National Science Foundation under Grant No. PHY9311610.
[1] D. DeMille, Phys. Rev. Lett. 74, 4165 (1995).

[2] S. G. Porsev, Y. G. Rakhlina, and M. G. Kozlov, Pis'ma Zh. Eksp. Teor. Fiz. 61, 449 (1995) [JETP Lett. 61, 459 (1995)].

[3] The $5 d 6 s^{3} D_{2}$ state may also be of interest for the study of atomic parity nonconservation. As noted by I. B. Khriplovich (private communication), a measurement of parity nonconservation in the $4 f^{14} 6 s^{2}{ }^{1} S_{0} \rightarrow 4 f^{14} 5 d 6 s^{3} D_{2}$ transition is sensitive only to nuclear-spin-dependent effects.

[4] M. A. Bouchiat et al., Phys. Lett. 117B, 358 (1982); 134, 463 (1984); J. Phys. 47, 1175 (1987).

[5] P. S. Drell and E. D. Commins, Phys. Rev. Lett. 53, 968 (1984); Phys. Rev. A 32, 2196 (1985).

[6] S. L. Gilbert and C. E. Wieman, Phys. Rev. A 34, 792 (1986); M. S. Noecker, B. P. Masterson, and C. E. Wieman, Phys. Rev. Lett. 61, 310 (1988).

[7] K. B. Blagoev and V. A. Komarovskii, At. Data Nucl. Data Tables 56, 1 (1994).

[8] D. Budker, E. D. Commins, D. DeMille, and M. S. Zolotorev, J. Opt. Soc. Am. 16, 1514 (1991); Phys. Rev. A 50, 132 (1994).

[9] The rate at which an atom makes a transition from an initial state $|i\rangle$ to a final state $|f\rangle$ in the presence of an electromagnetic field with frequency components $\omega_{1}$ and $\omega_{2}$ and corre- sponding electric-field polarization vectors $\vec{\varepsilon}_{1}$ and $\vec{\varepsilon}_{2}$ is given in [29]:

$$
I \propto\left|\sum_{n}\left[\frac{\left\langle f\left|\vec{\varepsilon}_{1} \cdot \overrightarrow{\mathbf{D}}\right| n\right\rangle\left\langle n\left|\vec{\varepsilon}_{2} \cdot \overrightarrow{\mathbf{D}}\right| i\right\rangle}{\omega_{n i}-\omega_{1}}+\frac{\left\langle f\left|\vec{\varepsilon}_{2} \cdot \overrightarrow{\mathbf{D}}\right| n\right\rangle\left\langle n\left|\vec{\varepsilon}_{1} \cdot \overrightarrow{\mathbf{D}}\right| i\right\rangle}{\omega_{n i}-\omega_{2}}\right]\right|^{2},
$$

where $|n\rangle$ is a complete set of intermediate states and $\omega_{n i}$ corresponds to the energy difference between the state $|n\rangle$ and the initial state. We define the two-photon operator as components of a $3 \times 3$ matrix

$$
\begin{gathered}
Q_{a b}=D_{a}\left(\sum_{n} \frac{|n\rangle\langle n|}{\omega_{n i}-\omega_{1}}\right) D_{b}+D_{b}\left(\sum_{n} \frac{|n\rangle\langle n|}{\omega_{n i}-\omega_{2}}\right) D_{a}, \\
I \propto\left|\varepsilon_{1 a} \varepsilon_{2 b}\left\langle f\left|Q_{a b}\right| i\right\rangle\right|^{2} .
\end{gathered}
$$

Here subscripts $a$ and $b$ refer to the Cartesian components of the electric-field polarization vectors and the electric-dipole operator $\overrightarrow{\mathbf{D}}$. $Q_{a b}$ can be decomposed as a sum of irreducible spherical tensors of rank 0,1 , and 2 . However, for a $J_{i}=0$ to $J_{f}=1$ transition, only the rank- 1 (vector) component can contribute to the matrix element. Noting that a rank-1 tensor is antisymmetric under exchange of indices,

$$
\begin{aligned}
Q_{a b} & =Q_{a b}^{(1)}=\frac{1}{2}\left(Q_{a b}-Q_{b a}\right) \\
& =\left(\omega_{1}-\omega_{2}\right)\left[\sum_{n} \frac{D_{a}|n\rangle\left\langle n\left|D_{b}-D_{b}\right| n\right\rangle\langle n| D_{a}}{\left(\omega_{n i}-\omega_{1}\right)\left(\omega_{n i}-\omega_{2}\right)}\right] .
\end{aligned}
$$


It is apparent that $Q_{a b}^{(1)}$ is zero when $\omega_{1}=\omega_{2}$. Therefore, one cannot excite a transition from $J_{i}=0$ to $J_{f}=1$ with a single frequency of light.

[10] W. C. Martin, R. Zalubas, and L. Hagan, Atomic Energy Levels - The Rare Earth Elements (National Bureau of Standards, Washington, D.C., 1978), and references therein.

[11] J.-F. Wyart and P. Camus, Phys. Scr. 20, 43 (1979).

[12] M. Baumann and G. Wandel, Phys. Lett. 22, 283 (1966).

[13] B. Budick and J. Snir, Phys. Rev. A 1, 545 (1970).

[14] W. Gornik et al., Opt. Commun. 6, 327 (1972).

[15] F. H. K. Rambow and L. O. Schearer, Phys. Rev. A 14, 738 (1976).

[16] M. L. Burshtein et al., Opt. Spektrosk. 37, 617 (1974) [Opt. Spectrosc. (USSR) 37, 351 (1974)].

[17] K. B. Blagoev, V. A. Komarovskii, and N. P. Penkin, Opt. Spectrosk. 45, 1024 (1978) [Opt. Spectrosc. (USSR) 45, 832 (1978)].

[18] M. Gustavsson, H. Lundberg, and L. Walsson, J. Opt. Soc. Am. 69, 984 (1979).

[19] J. E. Golub, Y. S. Bai, and T. W. Mossberg, Phys. Rev. A 37, 119 (1988).

[20] B. B. Konetskii, V. A. Mishin, and A. M. Prohorov, Zh. Prikl. Spektrosk. [J. Appl. Spectrosc. (USSR)] 54, 558 (1991); S. K. Borisov et al., Opt. Spektosk. 62, 1216 (1987) [Opt. Spectrosc. (USSR) 62, 719 (1987)].

[21] J. N. Dodd and G. W. Series, in Progress in Atomic Spectroscopy, Part A, edited by W. Hanle and H. Kleinpoppen (Plenum, New York, 1978).

[22] W.-G. Jin et al., J. Phys. Soc. Jpn. 60, 2896 (1991).

[23] The hyperfine splittings of the $5 d 6 s^{3} D_{1},{ }^{3} D_{2}$, and ${ }^{1} D_{2}$ states were estimated from previous measurements of the hyperfine splittings of the $6 s 6 d$ states. As shown in [30], the hyperfine structure of the fine-structure states represented in intermediate coupling can be parametrized in terms of single-particle effective radial integrals. The dominant contribution to the hyperfine structure in both $5 d 6 s$ and $6 s 6 d$ configurations comes from the $6 s$ electron. Using the effective radial integral of the $6 s$ electron derived from measurements of hyperfine splittings in the $6 s 6 d$ configuration [31], the hyperfine splittings in the $5 d 6 s$ configuration due to the $6 s$ electron were calculated and all were found to be greater than $200 \mathrm{MHz}$. The spin-orbit mixing coefficients of the $5 d 6 s$ configuration used in this calculation were derived [32] from the energy-level splittings between the $5 d 6 s$ fine-structure states [10].

[24] The amplitude of modulation of each hyperfine frequency component of the fluorescence signal from ${ }^{171} \mathrm{Yb}$ and ${ }^{173} \mathrm{Yb}$ was calculated using the density-matrix formalism [21]. The excited states populated according to the schemes of Figs. 2(b)-2(d) were all populated through $5 d 6 s^{3} D_{1}$ and ${ }^{1} D_{2}$. Offdiagonal elements of the density matrix between different hyperfine components of the $5 d 6 s$ states were set to zero since these components oscillate on time scales shorter than the duration of the excitation pulse. Linearly polarized light was used in each step of the excitation process and fluorescence light was detected without a polarizer. However, the direction of polarization was not well controlled. To account for this, the polarization of the excitation light and the detected light used in the calculation was varied in order to maximize the amplitude of modulation.

[25] W. Lange, J. Luther, and A. Stendel (unpublished).

[26] M. Baumann, M. Braun, A. Gaiser, and H. Liening, J. Phys. B 18, L601 (1985).

[27] Y. S. Bai and T. W. Mossberg, Phys. Rev. A 35, 619 (1987).

[28] I. I. Sobelman, Atomic Spectra and Radiative Transitions (Springer-Verlag, New York, 1992).

[29] W. Demtröder, Laser Spectroscopy (Springer-Verlag, Berlin, 1981).

[30] M. Gustavsson, G. Olsson, and A. Rosén, Z. Phys. A 290, 231 (1979).

[31] M. Baumann and M. Braun, Z. Phys. D 1, 247 (1985).

[32] E. U. Condon and G. H. Shortley, The Theory of Atomic Spectra (Cambridge University Press, Cambridge, 1957). 